\title{
NNLO mixed EW-QCD corrections to single vector boson production
}

\author{
Roberto Bonciani \\ Università di Roma "La Sapienza” and INFN Sezione di Romal \\ E-mail: roberto.bonciani@romal.infn.it

\section{Federico Buccioni} \\ Rudolf Peierls Centre for Theoretical Physics, Clarendon Laboratory, Parks Road, Oxford OX1 \\ $3 P U, U K$ \\ E-mail: federico.buccioniephysics.ox.ac.uk
}

\section{Narayan Rana*}

INFN Sezione di Milano, Via Celoria 16, 20133 Milano, Italy

E-mail: narayan.rana@mi.infn.it

\section{Alessandro Vicini}

Dipartimento di Fisica "Aldo Pontremoli”, University of Milano and INFN Sezione di Milano, Via Celoria 16, 20133 Milano, Italy

E-mail: alessandro.vicini@mi.infn.it

\begin{abstract}
We outline the computational details to obtain mixed EW-QCD corrections to on-shell production of a single vector boson at the LHC at two-loop level. We use the novel method of differential equation to obtain the pure virtual, real-virtual and double-real master integrals. Finally, we obtain the $\mathscr{O}\left(\alpha \alpha_{s}\right)$ corrections to the total partonic cross section of the process $q \bar{q} \rightarrow Z+X$.
\end{abstract}

14th International Symposium on Radiative Corrections (RADCOR2019)

9-13 September 2019

Palais des Papes, Avignon, France

${ }^{*}$ Speaker. 


\section{Introduction}

The Drell-Yan (DY) production of a pair of high transverse momentum leptons through the decay of a produced electrically neutral gauge boson $\left(\gamma^{*} / Z\right)$ at hadron colliders, is one of the most important processes for our understanding of Quantum Chromodynamics (QCD). Due to its clean signature and abundant production at the hadron colliders, it is used for the setting of several highprecision measurements of the electroweak (EW) sector of the Standard Model (SM), for instance, a precise measurement of the weak mixing angle and of the properties of the $Z$ boson. Because of its high importance, precise theoretical prediction for the $Z$ boson DY production has always gained much attention. Following the pioneering calculations of the next-to-leading order (NLO) [1] and next-to-next-to-leading order (NNLO) [2] QCD corrections to the total inclusive cross section, the fully differential description of the leptonic final state has been obtained in Refs. [3, 4, 5, 6]. Finally, the next-to-next-to-next-to-leading order $\left(\mathrm{N}^{3} \mathrm{LO}\right) \mathrm{QCD}$ corrections have been presented in the threshold limit, for both the total inclusive cross section [7, 8, 9] and rapidity distribution [10, 11]. While the QCD corrections have reached high precision, the NLO EW corrections, as shown in Refs. $[12,13,14,15]$, contribute at the $\mathscr{O}(1 \%)$ level as far as the total cross section is concerned and are comparable to that of the NNLO QCD contributions. Additionally in specific phase-space regions, kinematic distributions may be boosted, yielding corrections at the $\mathscr{O}(10 \%)$ level or more. Since control over the kinematic distributions in some cases is required at the per mille level for the high-precision determination of EW parameters (cf. Refs. [16, 17, 18] for specific cases), the computation of the mixed QCD-EW corrections is quite demanding, for both the study of the gauge boson resonances and of the high mass/momentum tails of the kinematic distributions [19, 20]. First steps have been taken by obtaining analytic expressions in Refs. [21, 22, 23, 24, 25]. They are then compared with the approximations available via Monte Carlo simulation tools [26, 27]. In these tools, the bulk of the leading effects, separately due to QCD and QED corrections, can be correctly evaluated for several observables, however, the remaining sub-leading QED effects and the genuine QCD-weak corrections are still missing, Additionally, the matching of factorizable QCD and EW contributions depends on the chosen recipes and hence introduces ambiguities to the estimation of theoretical uncertainties, which must be addressed. For these reasons the evaluation of the complete $\mathscr{O}\left(\alpha \alpha_{s}\right)$ corrections to the DY processes is very much desirable. In Refs. [28, 29, 30] the mixed QCD-QED corrections to the total inclusive cross section and transverse momentum spectrum of an on-shell $Z$ boson have been obtained. On another note, the Master Integrals (MIs) required to compute the complete QCD-EW mixed corrections to DY process has been presented in Refs. [31, 32].

In this proceeding, we discuss the results, presented in Ref. [33], for the total inclusive cross section of production of an on-shell $Z$ boson in the quark-antiquark partonic channel, including the complete set of QCD-EW corrections of $\mathscr{O}\left(\alpha \alpha_{s}\right)$.

\section{Theoretical framework}

The total inclusive production cross section $\sigma_{t o t}$ of a $Z$ boson at hadron colliders $(p p \rightarrow Z+X)$ can 
be written as

$$
\sigma_{t o t}(\tau)=\sum_{i, j \in q, \bar{q}, g, \gamma} \int \mathrm{d} x_{1} \mathrm{~d} x_{2} \hat{f}_{i}\left(x_{1}\right) \hat{f}_{j}\left(x_{2}\right) \hat{\sigma}_{i j}(z)
$$

where $z=\frac{m_{Z}^{2}}{\hat{s}}$ and $\tau=\frac{m_{Z}^{2}}{S}$ are the ratio of the squared $Z$ boson mass, $m_{Z}$, with $\hat{s}$ and $S$, the partonic and hadronic center of mass energy squared, respectively. $\hat{s}$ and $S$ are related by $\hat{s}=x_{1} x_{2} S$ through the Bjorken momentum fractions $x_{1}, x_{2} . \hat{f}_{i}(x)$ is the bare parton density of the $i$-th incoming parton and $\hat{\sigma}_{i j}$ is the bare cross section of the partonic process $i j \rightarrow Z+X$. The sum over $i, j$ includes quarks $(q)$, antiquarks $(\bar{q})$, gluons $(g)$ and photons $(\gamma)$. In the SM, we have the following double expansion of the partonic cross section in the electromagnetic and strong coupling constants, $\alpha$ and $\alpha_{s}$, respectively:

$$
\hat{\sigma}_{i j}(z)=\sum_{m, n=0}^{\infty} \alpha_{s}^{m} \alpha^{n} \hat{\sigma}_{i j}^{(m, n)}(z)
$$

where $\hat{\sigma}_{i j}^{(m, n)}$ is the correction of $\mathscr{O}\left(\alpha_{s}^{m} \alpha^{n}\right)$ to the lowest-order inclusive total cross section $\hat{\sigma}_{i j}^{(0,0)}$ of the partonic scattering $i j \rightarrow Z$. We consider the $q \bar{q}$ initiated scattering, specifically the case of an up-type quark: $q \bar{q}=u \bar{u}$. The following scattering processes contribute to the complete set of $\mathscr{O}\left(\alpha \alpha_{s}\right)$ corrections to $\hat{\sigma}_{u \bar{u}}$ :

$$
\begin{aligned}
& u \bar{u} \rightarrow Z, \\
& u \bar{u} \rightarrow Z g, \\
& u \bar{u} \rightarrow Z \gamma, \\
& u \bar{u} \rightarrow Z g \gamma, \\
& u \bar{u} \rightarrow Z u \bar{u}, \\
& u \bar{u} \rightarrow Z d \bar{d},
\end{aligned}
$$

where $d$ is a down-type massless quark. Results for the process (2.6) and QCD-QED contributions to process (2.3) have been presented in Ref. [34] and Ref. [21, 35], respectively. The corresponding results for $d \bar{d}$ initiated subprocesses can be obtained from our results with proper replacements of the electric charge $\left(Q_{f}\right)$ and the third component of the weak isospin $\left(I_{f}^{(3)}\right)$, for a fermion $f$.

The process (2.3) receives contributions (as shown in Fig. 1) from two-loop $2 \rightarrow 1$ Feynman diagrams interfered with the Born process (the double-virtual contributions), and interference of one-loop $2 \rightarrow 1$ Feynman diagrams (the virtual-virtual contributions).
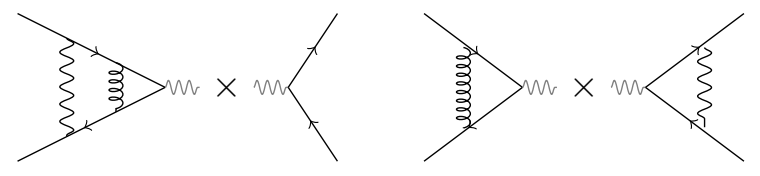

Figure 1: Schematic diagrams for double-virtual and virtual-virtual contributions.

The processes (2.4)-(2.5) receive contributions from one-loop $2 \rightarrow 2$ Feynman diagrams that have to be interfered with the corresponding tree-level (as schematically presented in Fig. 2). We refer them as the real-virtual contributions. 

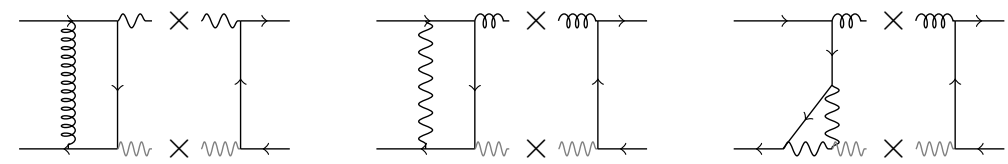

Figure 2: Schematic diagrams for the real-virtual contribution.

The last three processes, (2.6)-(2.8), receive contributions from tree-level $2 \rightarrow 3$ Feynman diagrams interfered with themselves (the double-real contributions as shown in Fig. 3).
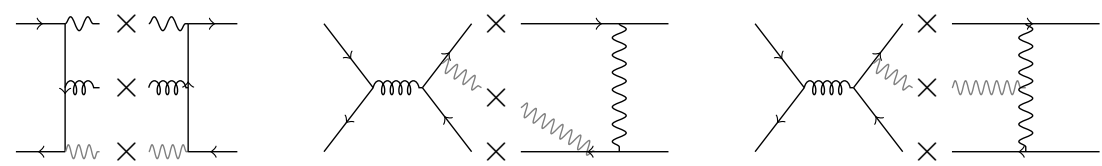

Figure 3: Schematic diagrams for the double-real contributions

The complete $\mathscr{O}\left(\alpha \alpha_{s}\right)$ corrections can be organized in two subsets, both gauge invariant: QCD-QED and QCD-weak contributions. Processes (2.3)-(2.7) contribute to the former, and processes (2.3), (2.4), (2.7) and (2.8) to the latter. On the other hand, depending on the presence of one real or virtual photon, of one virtual $Z$ boson, or of one/two virtual $W$ bosons, we can organize the full corrections in three groups. We note that the last two groups are separately gauge invariant. Furthermore, we do not include the processes with the emission of one extra massive on-shell gauge boson, as their measurement depends on the details of the experimental event selection.

\section{Computational details}

We follow the diagrammatic approach to obtain all the relevant contributions. We treat all the processes, virtual and real emission contributions, with the same algorithmic approach. Two independent parallel computations have been performed to have consistent check on our results. In one process, we obtain all the Feynman diagrams contributing to a given amplitude with QGRAF [36] and in another we use FeynArts [37]. Next, we perform algebraic simplifications using in-house FORM [38] and Mathematica routines. The scalar Feynman integrals are reduced to MIs using integration-by-parts (IBP) [39, 40, 41] and Lorentz-invariance (LI) identities [42]. The reduction procedure is performed using LiteRed $[43,44]$ in the first procedure and using Kira [45] and Reduze $2[46,47]$ in the second. The entire computation is carried out within dimensional regularization in $D=4-2 \varepsilon$ space-time dimensions. Then, we use the method of differential equations [48, 49, 42, 50, 51, 52, 53] to obtain the MIs, for both the pure virtual and real emission corrections. In the latter case, the phase-space delta functions are dealt using the reverse unitarity technique [54, 55], as follows

$$
\delta\left(p^{2}-m^{2}\right) \rightarrow \frac{1}{2 \pi i}\left(\frac{1}{p^{2}-m^{2}+i \eta}-\frac{1}{p^{2}-m^{2}-i \eta}\right) .
$$

Thus we transform the integration over the full phase space of the additional parton/s for processes (2.4)-(2.8), into the evaluation of the two-loop integrals with cut propagators. Apart from imposing an on-shell condition on the lines that correspond to the final-state particles, the integrals behave as 
loop integrals and hence one can use the techniques like IBP and method of differential equations to solve them. Below, we present a short description of the method (See [53, 56, 57] for details).

The MIs are functions of the space-time dimension $D$ and the variable $z$. The basic idea is to obtain a set of differential equations of the MIs by performing differentiation w.r.t $z$ and then to use the IBP identities. In all the cases in hand, we obtain a system of differential equations which can be organized in a block-triangular form, with most of the blocks being $1 \times 1$ and the rest being $2 \times 2$. For the coupled sub-systems, we obtain a second order linear differential equation by uncoupling and solve them using the method of variation of constant. The solution for each integral is obtained in series expansion in $\varepsilon$ up to required order. In calculating the MIs, the package HarmonicSums $[58,59,60]$ has been used.

The pure virtual MIs are presented in [61, 62, 63, 64, 65, 66], considering an off-shell $Z$ boson. In case of a single internal massive line, the solutions for these integrals contain generalised harmonic polylogarithms (GPLs) or harmonic polylogarithms (HPLs) [67, 68, 69, 70] over the alphabet

$$
\left\{\frac{1}{z}, \frac{1}{1-z}, \frac{1}{1+z}\right\}
$$

In case of two internal massive lines, square-root letters appear. To rationalize them, we introduce the Landau variable $x$ as

$$
\frac{\hat{s}}{m_{Z}^{2}}=-\frac{(1-x)^{2}}{x} .
$$

and the new letters contributing to the alphabet are

$$
\left\{\frac{1}{1-x+x^{2}}, \frac{x}{1-x+x^{2}}\right\}
$$

which are the cyclotomic extension [71] of the HPLs. Since in our case the $Z$ boson is on-shell, we have obtained these integrals taking the appropriate on-shell limit, i.e. evaluating the GPLs or HPLs at $z=1$ or equivalent limit of $x$. All the constants appearing, can be reduced to the basis introduced in Ref. [72]. The off-shell integrals and most of their on-shell limit have been checked using FIESTA $[73,74,75]$.

The two- and three-body phase-space MIs with only gluon or photon lines are already available in the literature [55]. To validate our routines developed for the present calculation, however, we have recomputed them and found complete agreement with the known expressions. We have computed all the new MIs, with one or two internal massive lines. During the computation, we need to introduce several other variables e.g. $w$ and $\rho$ as

$$
z=\frac{w}{(1+w)^{2}} ; \quad z=\frac{\rho}{1-\rho+\rho^{2}}
$$

to obtain a rationalized alphabet with addition of the following letters

$$
\left\{\frac{1}{1+x+x^{2}}, \frac{x}{1+x+x^{2}}, \frac{1}{1+x^{2}}, \frac{x}{1+x^{2}}\right\} .
$$

The boundary conditions are obtained by explicitly calculating the MIs in the soft limit $(z \rightarrow 1)$.

After the phase-space integration, the various contributions to partonic total cross section depends solely on the variable $z$. The virtual contributions are proportional to $\delta(1-z)$. The part that 
corresponds to processes (2.4)-(2.8) is expressed almost entirely in terms of $\delta(1-z)$ and of GPLs, or cyclotomic HPLs [71], functions of $z$. Three MIs appearing in processes (2.7) and (2.8) satisfy elliptic differential equations, whose homogeneous behaviour has already been studied in Ref. [64]. We have obtained their complete solution with a series expansion around $z=1$ (see for instance $[76,64,77,78,79,80,81])$. In the computation of the MIs, the mass of the $W$ boson is set equal to $m_{Z}$, the mass of $Z$ boson, to avoid the presence of an additional energy scale in the problem, which would make the analytical solution of the differential equations in terms of known functions more complicated. However, to obtain precise result, we can perform an expansion of the integrand in powers of the ratio $\delta_{m}^{2}=\left(m_{Z}^{2}-m_{W}^{2}\right) / m_{Z}^{2}$, and reduce all the terms of the series to a combination of the same basic equal-mass MIs. We note that the couplings of the $Z$ boson to fermions are expressed in terms of the physical value of the weak mixing angle $\sin ^{2} \theta_{W}=1-m_{W}^{2} / m_{Z}^{2}$.

\section{Ultraviolet and infrared singularities}

In general, we require the renormalization of the couplings and the fields up to $\mathscr{O}\left(\alpha \alpha_{s}\right)$. However, the Born process does not contain $\alpha_{s}$, hence no ultraviolet (UV) renormalization is required for the same. The $Z$ boson field and the EW couplings also do not receive $\mathscr{O}\left(\alpha_{s}\right)$ renormalization corrections. On the other hand, the quark field receives EW $(\mathscr{O}(\alpha))$ and mixed QCD-EW $\left(\mathscr{O}\left(\alpha \alpha_{s}\right)\right)$ renormalization corrections which we consider in the on-shell scheme. To simplify the UV singularities arising from the EW sector, we perform the calculation in the EW background field gauge (BFG) [82]. The advantage of using BFG is that, on the one hand, the combination of one-particle-irreducible vertex and external quark wave function corrections are UV finite, and on the other, the external $Z$ boson wave function and the lowest-order coupling renormalization corrections, whose combination, order-by-order in perturbation theory, is also UV finite. We remark that, in general notion, the EW gauge sector of the SM Lagrangian depends on three parameters $\left(g, g^{\prime}, v\right)$, the two gauge couplings and the Higgs-doublet vacuum expectation value. However, we choose to use the combination of $\left(G_{\mu}, m_{W}, m_{Z}\right)$, respectively the Fermi constant, the $W$ and $Z$ boson masses, after introducing counterterms and renormalized parameters. See Ref. [83] for the description of the additional counterterms appearing for such replacement. An alternative scheme with the effective leptonic weak mixing angle as input parameter has been discussed in Ref. [84].

The infrared (IR) singularities arising in this scenario are of two types by nature: one is called soft singularity due to a soft massless boson and the other is collinear singularity coming from collinear partons. As the $\mathscr{O}\left(\alpha \alpha_{s}\right)$ corrections are organized in two gauge invariant subsets: QCDQED and QCD-weak contributions, we study the IR singularities for each subset. For both the subsets, once all the degenerate states are summed up, i.e. the processes (2.3)-(2.8), the soft and final-state collinear singularities cancel. What remains are the collinear singularities arising from initial states, which are removed by mass factorization. For the QCD-QED subset, a new type of mass factorization kernel $\Gamma_{i j}$ with mixed non-factorizing contributions, appears [85]. However initial state collinear singularities in the QCD-weak case are of QCD origin only. The mass factorization introduces the physical parton densities $f_{i}\left(x, \mu_{F}\right)$, at the factorization scale $\mu_{F}$, which are defined through the mass factorization kernel $\Gamma_{i j}$ as follows

$$
\hat{f}_{i}=f_{j} \otimes \Gamma_{i j} .
$$


The kernel admits series expansion in $\alpha$ and $\alpha_{s}$. Finally, using Eq. (4.1) in Eq. (2.1), we obtain the total cross section expressed in terms of subtracted, finite, partonic cross sections $\sigma_{i j}\left(z, \mu_{F}\right)$ :

$$
\sigma_{t o t}(z)=\sum_{i, j \in q, \bar{q}, g, \gamma} \int_{\gamma} \mathrm{d} x_{1} \mathrm{~d} x_{2} f_{i}\left(x_{1}, \mu_{F}\right) f_{j}\left(x_{2}, \mu_{F}\right) \sigma_{i j}\left(z, \mu_{F}\right) .
$$

The $\sigma_{i j}$ also admits a perturbative expansion in powers of $\alpha$ and $\alpha_{s}$, in analogy to Eq. (2.2).

\section{Results}

We obtain the results for $\sigma_{u \bar{u}}^{(1,1)}$. As mentioned earlier, $\sigma_{u \bar{u}}^{(1,1)}$ depends on $z$ through $\delta(1-z)$ and GPLs [67, 68, 69, 70] or cyclotomic HPLs [71]. Additionally, the contributions from elliptic integrals appear as series expansion around threshold i.e. $z \rightarrow 1$. With the $\delta(1-z)$ contribution, apart from the multiple zeta values [86], the following constants appear

$$
\left\{\ln 2, \operatorname{Li}_{4}(1 / 2), \mathrm{GI}\left[r_{2}\right], \mathrm{GI}\left[0, r_{2}\right], \mathrm{GI}\left[0,1, r_{4}\right]\right\},
$$

where $r_{i}$ are the sixth root of unity defined as

$$
r_{1}=e^{i \pi / 3}, r_{2}=e^{-i \pi / 3}, r_{3}=e^{2 i \pi / 3}, r_{4}=e^{-2 i \pi / 3} .
$$

Additionally, the computation of the boundary constants of the differential equations for the doublereal emissions, generates the following cyclotomic constants [71, 87]

$$
\begin{aligned}
& H\left[\{4,1\}, 0,-1,(-1)^{2 / 3}\right], H\left[\{4,1\}, 0,0,(-1)^{2 / 3}\right], H\left[\{4,1\},\{3,0\},-1,(-1)^{2 / 3}\right], \\
& H\left[\{4,1\},\{3,1\},-1,(-1)^{2 / 3}\right], H\left[\{4,1\},\{3,0\}, 0,(-1)^{2 / 3}\right], H\left[\{4,1\},\{3,1\}, 0,(-1)^{2 / 3}\right] .
\end{aligned}
$$

Here, the letters of cyclotomy 3 and 4 are

$$
f_{\{3,0\}}(x)=\frac{1}{1+x+x^{2}}, f_{\{3,1\}}(x)=\frac{x}{1+x+x^{2}}, f_{\{4,0\}}(x)=\frac{1}{1+x^{2}}, f_{\{4,1\}}(x)=\frac{x}{1+x^{2}} .
$$

In order to anticipate the relative size of the different sets of corrections, we define:

$$
\alpha_{s} \alpha \sigma_{u \bar{u}}^{(1,1)}=\sigma_{u \bar{u}}^{(0)}\left(\Delta_{u \bar{u}, \gamma}^{(1,1)}+\Delta_{u \bar{u}, Z}^{(1,1)}+\Delta_{u \bar{u}, W}^{(1,1)}\right)
$$

where $\sigma_{u \bar{u}}^{(0,0)} \equiv \sigma_{u \bar{u}}^{(0)} \delta(1-z)=4 \sqrt{2} G_{\mu}\left(\pi / N_{c}\right)\left(C_{v, u}^{2}+C_{a, u}^{2}\right) \delta(1-z)$ is the Born cross section. $N_{c}$ is the number of colours and $C_{v / a, u}$ are the vector/axial-vector couplings of the $Z$ boson to the up quark. $\Delta_{u \bar{u}, K}^{(1,1)}$ with $K=\gamma, Z, W$ are the corrections due to the exchange of a photon, a $Z$ boson, and of one or two $W$ boson/s including the lowest order charge renormalization counterterms, respectively. We introduce the NLO-QCD correction to the same partonic process, defined as $\alpha_{s} \sigma_{u \bar{u}}^{(1,0)}=\sigma_{u \bar{u}}^{(0)} \Delta_{u \bar{u}}^{(1,0)}$, to have a comparison. In Figure 4 we present the contributions of the different subsets, $\Delta_{u \bar{u}, K}^{(1,1)}$ with $K=\gamma, Z, W$, and their sum. We also present $\Delta_{u \bar{u}}^{(1,0)}$, divided by a factor 10 . We exclude from the plot all the contributions proportional to $\delta(1-z)$. For the numerics, we use the following input parameters:

\begin{tabular}{ll}
\hline \hline$m_{W}=80.385 \mathrm{GeV}$ & $m_{Z}=91.1876 \mathrm{GeV}$ \\
$m_{t}=173.5 \mathrm{GeV}$ & $m_{H}=125 \mathrm{GeV}$ \\
$G_{\mu}=1.1663781 \times 10^{-5} \mathrm{GeV}^{-2}$ & $\alpha_{s}\left(m_{Z}\right)=0.118$ \\
\hline \hline
\end{tabular}

$m_{t}$ and $m_{H}$ are the top quark and Higgs boson mass, respectively. We set the factorisation scale $\mu_{F}=m_{Z}$. 


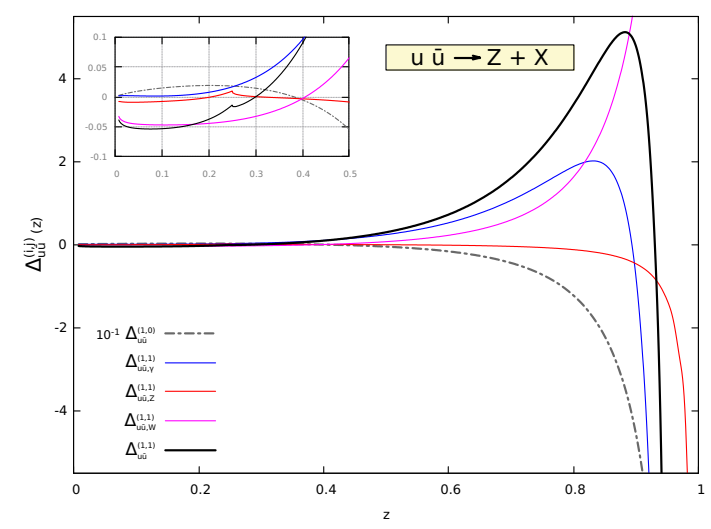

Figure 4: $\Delta_{u \bar{u}}^{(1,0)}$ in grey dashed, $\Delta_{u \bar{u}, K}^{(1,1)}$ with $K=\gamma, Z, W$ in blue, red, and magenta, respectively, and their sum in black solid, as a function of the partonic variable $z \cdot \Delta_{u \bar{u}}^{(1,0)}$ is divided by a factor 10 .

\section{Conclusion}

In this proceeding, we discuss Ref. [33], where we have presented the first results for the total inclusive partonic cross section for the process $q \bar{q} \rightarrow Z+X$, including the exact $\mathscr{O}\left(\alpha \alpha_{s}\right)$ corrections, with both photon and $W / Z$ boson exchanges. The results are analytic and are expressed in terms of GPLs, but also contain three elliptic MIs, which have been computed with a series expansion around $z=1$. The universal structure of the infrared singularities along with the final cancellation among all sub-processes to produce a subtracted, finite, partonic cross section, provides also a strong check on our calculation. The computation represents an important step towards the evaluation of the hadron-level cross section for $Z$ production at this perturbative order.

\section{Acknowledgments}

N.R. thanks the CERN Theory Department for hospitality and support during the completion of this work. A.V. is supported by the European Research Council under the European Unions Horizon 2020 research and innovation Programme (grant agreement number 740006). F.B. warmly thanks the Physics Institute of the University of Zurich where large part of this work was carried out and acknowledges support from the Swiss National Science Foundation (SNF) under contract BSCGI0-157722. The research of F.B. was partially supported by the ERC Starting Grant 804394 HIPQCD. R.B. and N.R. acknowledge the COST (European Cooperation in Science and Technology) Action CA16201 PARTICLEFACE for partial support.

\section{References}

[1] G. Altarelli, R. K. Ellis and G. Martinelli, Large Perturbative Corrections to the Drell-Yan Process in QCD, Nucl. Phys. B157 (1979) 461.

[2] R. Hamberg, W. van Neerven and T. Matsuura, A Complete calculation of the order $\alpha-s^{2}$ correction to the Drell-Yan K factor, Nucl.Phys. B359 (1991) 343. 
[3] C. Anastasiou, L. J. Dixon, K. Melnikov and F. Petriello, High precision QCD at hadron colliders: Electroweak gauge boson rapidity distributions at NNLO, Phys. Rev. D69 (2004) 094008 [hep-ph/0312266].

[4] K. Melnikov and F. Petriello, Electroweak gauge boson production at hadron colliders through O(alpha(s)**2), Phys. Rev. D74 (2006) 114017 [hep-ph/ 0609070 ].

[5] S. Catani, L. Cieri, G. Ferrera, D. de Florian and M. Grazzini, Vector boson production at hadron colliders: a fully exclusive QCD calculation at NNLO, Phys. Rev. Lett. 103 (2009) 082001 [0903.2120].

[6] R. Gavin, Y. Li, F. Petriello and S. Quackenbush, FEWZ 2.0: A code for hadronic Z production at next-to-next-to-leading order, Comput. Phys. Commun. 182 (2011) 2388 [1 011.3540 ].

[7] T. Ahmed, M. Mahakhud, N. Rana and V. Ravindran, Drell-Yan Production at Threshold to Third Order in QCD, Phys. Rev. Lett. 113 (2014) 112002 [1404.0366].

[8] Y. Li, A. von Manteuffel, R. M. Schabinger and H. X. Zhu, $N^{3}$ LO Higgs and Drell-Yan production at threshold: the one-loop two-emission contribution, 1404.5839.

[9] S. Catani, L. Cieri, D. de Florian, G. Ferrera and M. Grazzini, Threshold resummation at $N^{3} L L$ accuracy and soft-virtual cross sections at $N^{3}$ LO, Nucl. Phys. B888 (2014) 75 [1 405 . 4827].

[10] T. Ahmed, M. K. Mandal, N. Rana and V. Ravindran, Rapidity Distributions in Drell-Yan and Higgs Productions at Threshold to Third Order in QCD, Phys. Rev. Lett. 113 (2014) 212003 [1404.650 4].

[11] G. Lustermans, J. K. L. Michel and F. J. Tackmann, Generalized Threshold Factorization with Full Collinear Dynamics, 1908.00985.

[12] U. Baur, O. Brein, W. Hollik, C. Schappacher and D. Wackeroth, Electroweak radiative corrections to neutral current Drell-Yan processes at hadron colliders, Phys.Rev. D65 (2002) 033007 [hep-ph/0108274].

[13] C. Carloni Calame, G. Montagna, O. Nicrosini and A. Vicini, Precision electroweak calculation of the production of a high transverse-momentum lepton pair at hadron colliders, JHEP 0710 (2007) 109 [0710.1722].

[14] A. Arbuzov, D. Bardin, S. Bondarenko, P. Christova, L. Kalinovskaya et al., One-loop corrections to the Drell-Yan process in SANC. (II). The Neutral current case, Eur.Phys.J. C54 (2008) 451 [0711.0625].

[15] S. Dittmaier and M. Huber, Radiative corrections to the neutral-current Drell-Yan process in the Standard Model and its minimal supersymmetric extension, JHEP 01 (2010) 060 [0911.2329].

[16] S. Alioli et al., Precision studies of observables in $p p \rightarrow W \rightarrow l v_{l}$ and $p p \rightarrow \gamma, Z \rightarrow l^{+} l^{-}$processes at the LHC, Eur. Phys. J. C77 (2017) 280 [1606.02330].

[17] C. M. Carloni Calame, M. Chiesa, H. Martinez, G. Montagna, O. Nicrosini, F. Piccinini et al., Precision Measurement of the W-Boson Mass: Theoretical Contributions and Uncertainties, Phys. Rev. D96 (2017) 093005 [1612.02841].

[18] G. Bozzi, L. Citelli and A. Vicini, Parton density function uncertainties on the W boson mass measurement from the lepton transverse momentum distribution, Phys. Rev. D91 (2015) 113005 [1501.05587].

[19] G. Balossini, G. Montagna, C. M. Carloni Calame, M. Moretti, M. Treccani, O. Nicrosini et al., Electroweak \& QCD corrections to Drell Yan processes, Acta Phys. Polon. B39 (2008) 1675 [0805.1129]. 
[20] G. Balossini, G. Montagna, C. M. Carloni Calame, M. Moretti, O. Nicrosini, F. Piccinini et al., Combination of electroweak and QCD corrections to single W production at the Fermilab Tevatron and the CERN LHC, JHEP 01 (2010) 013 [0907 .0276].

[21] W. B. Kilgore and C. Sturm, Two-Loop Virtual Corrections to Drell-Yan Production at order $\alpha_{s} \alpha^{3}$, Phys. Rev. D85 (2012) 033005 [1107.4798].

[22] S. Dittmaier, A. Huss and C. Schwinn, Mixed QCD-electroweak $\mathscr{O}\left(\alpha_{s} \alpha\right)$ corrections to Drell-Yan processes in the resonance region: pole approximation and non-factorizable corrections, Nucl.Phys. B885 (2014) 318 [1403.3216].

[23] S. Dittmaier, A. Huss and C. Schwinn, $\mathscr{O}\left(\alpha_{s} \alpha\right)$ corrections to Drell-Yan processes in the resonance region, PoS LL2014 (2014) 045 [1 405 . 68 97].

[24] S. Dittmaier, A. Huss and C. Schwinn, Dominant mixed QCD-electroweak $\mathscr{O}\left(\alpha_{s}\right)$ corrections to Drell-Yan processes in the resonance region, Nucl. Phys. B904 (2016) 216 [1511. 08016 ].

[25] S. Dittmaier, A. Huss and C. Schwinn, Dominant $\mathscr{O}\left(\alpha_{s} \alpha\right)$ corrections to Drell-Yan processes in the resonance region, in Proceedings, 12th International Symposium on Radiative Corrections (Radcor 2015) and LoopFest XIV (Radiative Corrections for the LHC and Future Colliders), 2016, 1601.02027.

[26] L. Barze, G. Montagna, P. Nason, O. Nicrosini and F. Piccinini, Implementation of electroweak corrections in the POWHEG BOX: single W production, JHEP 04 (2012) 037 [1202. 0465 ].

[27] L. Barze, G. Montagna, P. Nason, O. Nicrosini, F. Piccinini and A. Vicini, Neutral current Drell-Yan with combined QCD and electroweak corrections in the POWHEG BOX, Eur. Phys. J. C73 (2013) $2474[1302.4606]$.

[28] D. de Florian, M. Der and I. Fabre, $Q C D \oplus Q E D$ NNLO corrections to Drell Yan production, Phys. Rev. D98 (2018) 094008 [1805.12214].

[29] L. Cieri, G. Ferrera and G. F. R. Sborlini, Combining QED and QCD transverse-momentum resummation for Z boson production at hadron colliders, JHEP 08 (2018) 165 [1805 . 11948 ].

[30] M. Delto, M. Jaquier, K. Melnikov and R. Roentsch, Mixed QCD $\otimes Q E D$ corrections to on-shell Z boson production at the LHC, 1909.08428.

[31] R. Bonciani, S. Di Vita, P. Mastrolia and U. Schubert, Two-Loop Master Integrals for the mixed EW-QCD virtual corrections to Drell-Yan scattering, JHEP 09 (2016) 091 [1604 . 08581 ].

[32] M. Heller, A. von Manteuffel and R. M. Schabinger, Multiple polylogarithms with algebraic arguments and the two-loop EW-QCD Drell-Yan master integrals, 1907.00491.

[33] R. Bonciani, F. Buccioni, N. Rana, I. Triscari and A. Vicini, NNLO $Q C D \times E W$ corrections to Z production in the qū channel, 1911.06200.

[34] R. Bonciani, F. Buccioni, R. Mondini and A. Vicini, Double-real corrections at $\mathscr{O}\left(\alpha \alpha_{s}\right)$ to single gauge boson production, Eur. Phys. J. C77 (2017) 187 [1611.00645].

[35] A. H. Ajjath, A. Chakraborty, P. K. Dhani, P. Mukherjee, N. Rana, V. Ravindran et al., NNLO $Q C D \oplus Q E D$ corrections to Higgs production in bottom quark annihilation, 1906.09028.

[36] P. Nogueira, Automatic Feynman graph generation, J.Comput.Phys. 105 (1993) 279.

[37] T. Hahn, Generating Feynman diagrams and amplitudes with FeynArts 3, Comput.Phys.Commun. 140 (2001) 418 [hep-ph/0012260]. 
[38] J. A. M. Vermaseren, New features of FORM, math-ph/0010025.

[39] F. Tkachov, A Theorem on Analytical Calculability of Four Loop Renormalization Group Functions, Phys.Lett. B100 (1981) 65.

[40] K. Chetyrkin and F. Tkachov, Integration by Parts: The Algorithm to Calculate beta Functions in 4 Loops, Nucl.Phys. B192 (1981) 159.

[41] S. Laporta, High precision calculation of multiloop Feynman integrals by difference equations, Int.J.Mod.Phys. A15 (2000) 5087 [hep-ph/ 0102033$].$

[42] T. Gehrmann and E. Remiddi, Differential equations for two loop four point functions, Nucl.Phys. B580 (2000) 485 [hep-ph/ 9912329 ].

[43] R. N. Lee, Presenting LiteRed: a tool for the Loop InTEgrals REDuction, 1212.2685.

[44] R. N. Lee, LiteRed 1.4: a powerful tool for reduction of multiloop integrals, J. Phys. Conf. Ser. 523 (2014) 012059 [1310.1145].

[45] P. Maierhoefer, J. Usovitsch and P. Uwer, Kira - A Feynman Integral Reduction Program, 1705.05610.

[46] C. Studerus, Reduze-Feynman Integral Reduction in C++, Comput.Phys.Commun. 181 (2010) 1293 [0912.2546].

[47] A. von Manteuffel and C. Studerus, Reduze 2 - Distributed Feynman Integral Reduction, 1201.4330 .

[48] A. Kotikov, Differential equations method: New technique for massive Feynman diagrams calculation, Phys.Lett. B254 (1991) 158.

[49] E. Remiddi, Differential equations for Feynman graph amplitudes, Nuovo Cim. A110 (1997) 1435 [hep-th/9711188].

[50] M. Argeri and P. Mastrolia, Feynman Diagrams and Differential Equations, Int.J.Mod.Phys. A22 (2007) 4375 [0707.4037].

[51] J. M. Henn, Lectures on differential equations for Feynman integrals, J. Phys. A48 (2015) 153001 [1412.2296].

[52] J. Ablinger, A. Behring, J. Blümlein, A. De Freitas, A. von Manteuffel and C. Schneider, Calculating Three Loop Ladder and V-Topologies for Massive Operator Matrix Elements by Computer Algebra, Comput. Phys. Commun. 202 (2016) 33 [1509.08324].

[53] J. Ablinger, J. Blümlein, P. Marquard, N. Rana and C. Schneider, Automated Solution of First Order Factorizable Systems of Differential Equations in One Variable, Nucl. Phys. B939 (2019) 253 [1810.12261].

[54] C. Anastasiou and K. Melnikov, Higgs boson production at hadron colliders in NNLO QCD, Nucl. Phys. B646 (2002) 220 [hep-ph/ 0207004 ].

[55] C. Anastasiou, S. Buehler, C. Duhr and F. Herzog, NNLO phase space master integrals for two-to-one inclusive cross sections in dimensional regularization, JHEP 11 (2012) 062 [1208 . 3130].

[56] J. Blümlein and C. Schneider, Analytic computing methods for precision calculations in quantum field theory, Int. J. Mod. Phys. A33 (2018) 1830015 [1809.02889].

[57] J. Ablinger, A. Behring, J. Blümlein, G. Falcioni, A. De Freitas, P. Marquard et al., Heavy quark form factors at two loops, Phys. Rev. D97 (2018) 094022 [1712.09889]. 
[58] J. Ablinger, A Computer Algebra Toolbox for Harmonic Sums Related to Particle Physics, Ph.D. thesis, Linz U., 2009. 1011.1176.

[59] J. Ablinger, J. Blümlein and C. Schneider, Analytic and Algorithmic Aspects of Generalized Harmonic Sums and Polylogarithms, J. Math. Phys. 54 (2013) 082301 [1302 . 0378].

[60] J. Ablinger, The package HarmonicSums: Computer Algebra and Analytic aspects of Nested Sums, PoS LL2014 (2014) 019 [1407. 6180].

[61] U. Aglietti and R. Bonciani, Master integrals with one massive propagator for the two loop electroweak form-factor, Nucl. Phys. B668 (2003) 3 [hep-ph/ 0304028 ].

[62] U. Aglietti and R. Bonciani, Master integrals with 2 and 3 massive propagators for the 2 loop electroweak form-factor - planar case, Nucl. Phys. B698 (2004) 277 [hep-ph/ 0401193 ].

[63] U. Aglietti, R. Bonciani, G. Degrassi and A. Vicini, Master integrals for the two-loop light fermion contributions to $\mathrm{gg} \longrightarrow \mathrm{H}$ and $\mathrm{H} \longrightarrow$ gamma gamma, Phys. Lett. B600 (2004) 57 [hep-ph/0407162].

[64] U. Aglietti, R. Bonciani, L. Grassi and E. Remiddi, The Two loop crossed ladder vertex diagram with two massive exchanges, Nucl. Phys. B789 (2008) 45 [0 705.2616$].$

[65] R. Bonciani, G. Degrassi and A. Vicini, On the Generalized Harmonic Polylogarithms of One Complex Variable, Comput. Phys. Commun. 182 (2011) 1253 [1007.1891].

[66] A. Kotikov, J. H. Kuhn and O. Veretin, Two-Loop Formfactors in Theories with Mass Gap and Z-Boson Production, Nucl. Phys. B788 (2008) 47 [hep-ph/ 0703013 ].

[67] A. Goncharov, Polylogarithms in arithmetic and geometry, Proceedings of the International Congress of Mathematicians 1,2 (1995) 374.

[68] A. B. Goncharov, Multiple polylogarithms and mixed tate motives, mat h/ 0103059.

[69] E. Remiddi and J. Vermaseren, Harmonic polylogarithms, Int.J.Mod.Phys. A15 (2000) 725 [hep-ph/9905237].

[70] J. Vollinga and S. Weinzierl, Numerical evaluation of multiple polylogarithms, Comput.Phys.Commun. 167 (2005) 177 [hep-ph/ 0410259 ].

[71] J. Ablinger, J. Blümlein and C. Schneider, Harmonic Sums and Polylogarithms Generated by Cyclotomic Polynomials, J. Math. Phys. 52 (2011) 102301 [1105. 6063 ].

[72] J. M. Henn, A. V. Smirnov and V. A. Smirnov, Evaluating Multiple Polylogarithm Values at Sixth Roots of Unity up to Weight Six, Nucl. Phys. B919 (2017) 315 [1512.08389].

[73] A. Smirnov and M. Tentyukov, Feynman Integral Evaluation by a Sector decomposiTion Approach (FIESTA), Comput.Phys.Commun. 180 (2009) 735 [0 807 . 4129].

[74] A. V. Smirnov, V. A. Smirnov and M. Tentyukov, FIESTA 2: Parallelizeable multiloop numerical calculations, Comput. Phys. Commun. 182 (2011) 790 [0912.0158].

[75] A. V. Smirnov, FIESTA4: Optimized Feynman integral calculations with GPU support, Comput. Phys. Commun. 204 (2016) 189 [1511.03614].

[76] S. Pozzorini and E. Remiddi, Precise numerical evaluation of the two loop sunrise graph master integrals in the equal mass case, Comput. Phys. Commun. 175 (2006) 381 [hep-ph/ 0505041 ].

[77] J. Blümlein and C. Schneider, The Method of Arbitrarily Large Moments to Calculate Single Scale Processes in Quantum Field Theory, Phys. Lett. B771 (2017) 31 [1701. 04614$].$ 
[78] R. N. Lee, A. V. Smirnov and V. A. Smirnov, Solving differential equations for Feynman integrals by expansions near singular points, JHEP 03 (2018) 008 [1709.07525].

[79] R. N. Lee, A. V. Smirnov and V. A. Smirnov, Evaluating elliptic master integrals at special kinematic values: using differential equations and their solutions via expansions near singular points, JHEP 07 (2018) 102 [1805.00227].

[80] R. Bonciani, G. Degrassi, P. P. Giardino and R. Groeber, A Numerical Routine for the Crossed Vertex Diagram with a Massive-Particle Loop, Comput. Phys. Commun. 241 (2019) 122 [1812. 02698 ].

[81] J. Blümlein, P. Marquard, N. Rana and C. Schneider, The Heavy Fermion Contributions to the Massive Three Loop Form Factors, Nucl. Phys. B949 (2019) 114751 [1908 . 00357 ].

[82] A. Denner, G. Weiglein and S. Dittmaier, Application of the background field method to the electroweak standard model, Nucl. Phys. B440 (1995) 95 [hep-ph/9410338].

[83] G. Degrassi and A. Vicini, Two loop renormalization of the electric charge in the standard model, Phys. Rev. D69 (2004) 073007 [hep-ph/ 0307122 ].

[84] M. Chiesa, F. Piccinini and A. Vicini, Direct determination of $\sin ^{2} \theta_{\text {eff }}^{\ell}$ at hadron colliders, Phys. Rev. D100 (2019) 071302 [1906.11569].

[85] D. de Florian, G. F. R. Sborlini and G. Rodrigo, QED corrections to the AltarelliâĂSPParisi splitting functions, Eur. Phys. J. C76 (2016) 282 [1512.00612].

[86] J. Blümlein, D. J. Broadhurst and J. A. M. Vermaseren, The Multiple Zeta Value Data Mine, Comput. Phys. Commun. 181 (2010) 582 [0907.2557].

[87] D. J. Broadhurst, Massive three - loop Feynman diagrams reducible to SC* primitives of algebras of the sixth root of unity, Eur. Phys. J. C8 (1999) 311 [hep-th/9803091]. 